\title{
REVISIÓN DESDE EL DESEMPEÑO LABORAL AL VÍNCULO CON ENFERMERÍA
}

\author{
A REVIEW FROM JOB PERFORMANCE TO THE LINK WITH NURSING
}

\author{
Andrés Jiménez Castro \\ Enfermero \\ Estudiante de Maestría en Administración de Enfermería \\ Facultad de Enfermería y Nutrición, Universidad Autónoma de San Luis Potosí, México \\ andres.mae.mx@gmail.com \\ https://orcid.org/0000-0002-9342-7374
}

\section{Sofia Echeverría Rivera}

Licenciada en Enfermería, Doctora en Administración

Maestra en Administración de Servicios de Enfermería, Facultad de Enfermería y Nutrición, Universidad Autónoma de San Luis Potosí, México

sofia@uaslp.mx

https://orcid.org/0000-0003-1099-8719

Artículo recibido el 18 de enero de 2021. Aceptado en versión corregida el 26 de junio de 2021.

\section{RESUMEN}

El recurso humano es el principal activo con el cual cuenta una institución pública o privada; esto se debe a que las organizaciones necesariamente están compuestas por personas, y sin ellas no podrían existir. Las corrientes modernas de administración en sistemas de salud están poniendo mayor atención al desempeño de las organizaciones, relacionándolas con el desempeño de su capital humano. OBJETIVO: Describir los conceptos y dimensiones del desempeño laboral planteado por diferentes autores en administración, mediante una revisión de literatura, y su relación con estudios realizados en enfermería. METODOLOGíA: Se realizó una búsqueda bibliográfica sistematizada sobre desempeño laboral en la Biblioteca Virtual Universitaria, en Centro de Recursos Académicos Informáticos Virtuales (CREATIVA), utilizando las bases de datos PubMed, Lilacs, Scielo y Scopus, en idioma portugués, inglés y español, entre los años 2010 y 2020. RESULTADOS: Se presentan dos áreas temáticas preestablecidas: La primera, es revisión de literatura en desempeño laboral y la segunda estudios en desempeño laboral de enfermería. Estas áreas se fueron relacionando con dimensiones teóricas del desempeño laboral. CONCLUSIÓN: Conocer el nivel el desempeño laboral del profesional de enfermería y las áreas que abarcan los conceptos teóricos en la literatura en administración y recurso humano, entrega mayores competencias, formación continua, y mayor profesionalismo (autonomía, comunicación y participación en 
Revisión desde el desempeño laboral al vínculo con Enfermería organizaciones sanitarias). De esta forma, ayuda a establecer estrategias de abordaje para conocer las falencias del desempeño en enfermería, realizar un análisis del contexto en donde se desarrolla el fenómeno en los profesionales y poder entregar una solución, mejorando el desempeño del profesional o del equipo de salud.

Palabras clave: revisión, desempeño, desempeño laboral, enfermería (DecS).

\begin{abstract}
The human resource is the main asset of a public or private institution; this is because organizations are necessarily made up of people, and without them, they could not exist. Modern management trends in health systems are paying greater attention to the performance of organizations, relating them to the performance of their human capital. OBJECTIVE: To describe the concepts and dimensions of work performance proposed by different authors in management, through a review of the literature, and their relationship with studies carried out in nursing. METHODOLOGY: A systematized bibliographic search on work performance was carried out in the Virtual University Library, in the Virtual Academic Computer Resource Centre (CREATIVA), using the databases PubMed, Lilacs, Scielo, and SCOPUS, in Portuguese, English and Spanish, between 2010 and 2020. RESULTS: two preestablished thematic areas are presented: the first is a review of the literature on work performance and the second studies on nursing work performance. These areas were related to theoretical dimensions of job performance. CONCLUSION: Knowing the level of nursing professional job performance and the areas covered by the theoretical concepts in the literature on administration and human resources, provides greater competencies, continuous training, and greater professionalism (autonomy, communication and participation in health organizations). In this way, it helps to establish strategies to address the shortcomings of nursing performance, to carry out an analysis of the context in which the phenomenon develops in professionals, and to be able to provide a solution, improving the performance of the professional or the health team.
\end{abstract}

Keywords: review, performance, work performance, nursing (DecS).

\title{
http://dx.doi.org/10.7764/Horiz_Enferm.32.2.177-191
}

\section{INTRODUCCIÓN}

El desempeño laboral está fuertemente asociado con el comportamiento de los integrantes de las organizaciones. Por ello, el buen desempeño individual de un colaborador, es una contribución positiva hacia el desempeño de la organización en cuanto al liderazgo y

empoderamiento. Los líderes pueden medir a través de diferentes mecanismos este desempeño, como lo plantea Saleem y Amin (2013). Por otro lado Choong (2014), menciona que las organizaciones de salud necesitan colaboradores con un alto desempeño laboral, para lograr los 
objetivos organizacionales y seguir siendo competitivos; a esto se suma lo encontrado en la literatura, donde se menciona que el rendimiento laboral depende de las cargas de trabajo, el tiempo de trabajo y la rentabilidad o beneficio económico que la organización le entrega al individuo ${ }^{(1)}$.

Por otro lado, el capital humano en organizaciones de salud, específicamente en enfermería es de suma relevancia, ya que equivale al $60 \%$ de la fuerza laboral en las organizaciones sanitarias. Mediante el informe 2020, elaborado por la Organización Mundial de la Salud (OMS) en colaboración con el Consejo Internacional de Enfermeras (CIE) y la campaña Nursing Now, revela que el personal de enfermería cuenta actualmente con cerca de 28 millones de profesionales en todo el mundo. Específicamente México contribuye con un capital humano de 312.347 profesionales de enfermería, según datos del Sistema de Información Administrativa de Recursos Humanos en Enfermería (SIARHE).

El desempeño del profesional de enfermería, se ve reflejado en la gestión de los cuidados entregados a los usuarios en los diferentes niveles de atención en salud. Al igual que en otras áreas de trabajo, se requiere hacer un análisis de conceptos, concepciones y enfoques, así ir adecuando a los profesionales de enfermería en nuevos paradigmas de la temática. En la actualidad, existe un gran interés por comprender el fenómeno de desempeño de los colaboradores en la organización, sin embargo, la nueva literatura científica en la temática, no aborda los avances conseguidos al interior de las instituciones de salud ${ }^{(1,2)}$.
El Objetivo de esta revisión, es describir los conceptos y dimensiones del desempeño laboral planteado por diferentes autores en administración, mediante una revisión de literatura, y su relación con estudios realizados en enfermería.

\section{METODOLOGÍA}

Se realizó una revisión de la literatura para responder al objetivo anteriormente planteado, reuniendo toda la evidencia empírica disponible, realizando una búsqueda de información en la base de datos BIG (búsqueda de información global) del campus creativa, perteneciente a la plataforma de biblioteca de la Universidad Autónoma de San Luis Potosí. Las bases de datos consultadas para obtener la literatura empleada en el diseño del contenido fueron ACSESS Digital Library, Biblioteca Virtual Miguel de Cervantes, Biblioteca Digital Mundial, Directory of Open Access Books y Bookshelf.

La revisión de artículos científicos fue en búsqueda de coincidencias y/o divergencias en estudios realizados sobre desempeño laboral en enfermería, utilizando la búsqueda de palabras clave que involucran las variables a investigar en la base de datos PubMed, Scopus, Scielo y LILACS. Luego fueron tomados en cuenta parámetros de inclusión, tales como: el idioma inglés, portugués y español, años de publicación entre 2010 al 2020 con interés en artículos relacionados con la temática. Los términos de búsqueda utilizados fueron: desempeño AND enfermería, desempeño OR laboral AND enfermería, work OR performance AND 
Revisión desde el desempeño laboral al vínculo con Enfermería

nursing, job OR performance AND nursing, todos ellos identificados previamente como términos Mesh o sus equivalentes DecS en español.

Se realizó un análisis de la información recabada (196 artículos), a través, de la lectura crítica del contenido. En primera instancia del título de las publicaciones, se descartaron las que no correspondían a la temática, de las publicaciones resultantes (133 artículos), se analizaron los resúmenes y de esta manera se realizó una segunda selección de publicaciones. Se volvió aplicar el análisis en los criterios de selección, en los textos completos y de esta manera resultaron 46 artículos en la selección que conformó la muestra final.

Finalmente, en la revisión sistemática se incluyeron 46 artículos (Figura 1).

Figura 1. Diagrama de flujo artículos seleccionados.

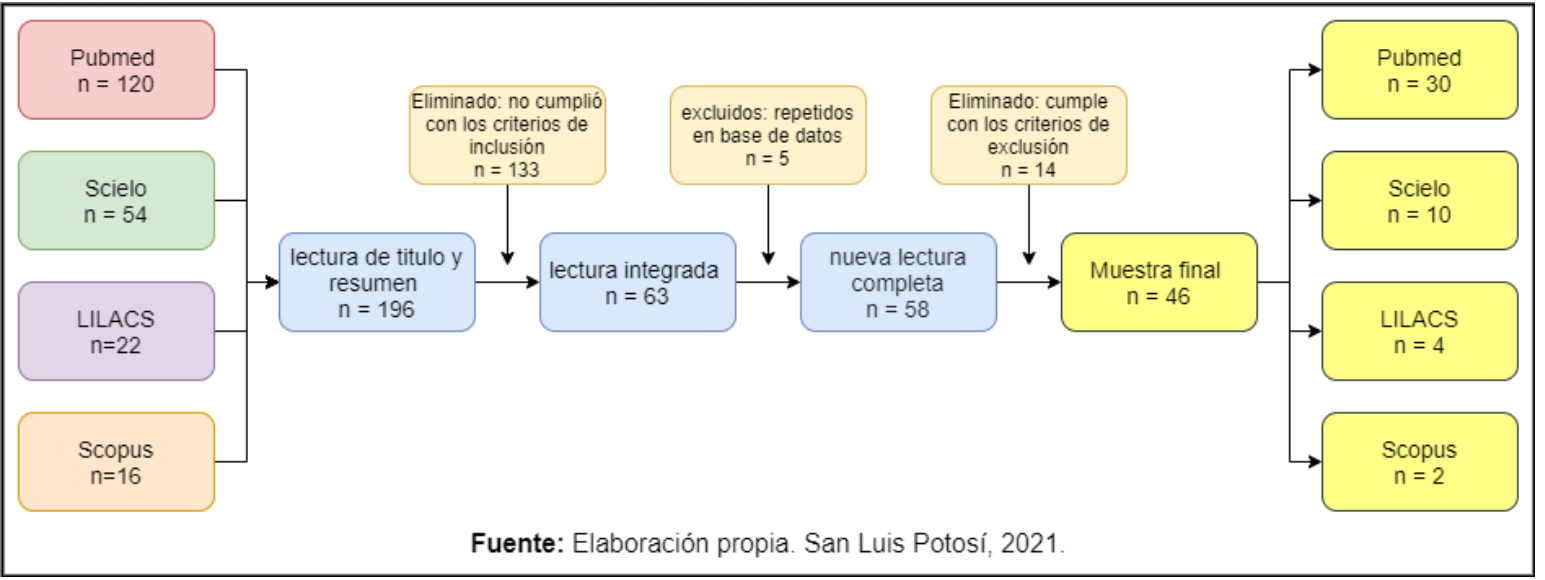

\section{RESULTADOS}

Los resultados se presentan en dos áreas temáticas preestablecidas: La primera (A), es revisión de literatura en desempeño laboral y la segunda (B) búsqueda de estudios en desempeño laboral de enfermería. Estas áreas se fueron relacionando con dimensiones teóricas del desempeño laboral, como lo son sus conceptos, fundamento, características, factores influyentes, elementos y evaluación del desempeño laboral.

\section{A. Revisión de literatura}

\section{Fundamento y concepto en desempeño laboral}

El desempeño laboral según Robbins (2004), describe que uno de los principios fundamentales es la psicología del desempeño y fijación de metas, la cual activa el comportamiento y mejora el desempeño, porque ayuda a la persona a enfocar sus esfuerzos sobre metas difíciles ${ }^{(3)}$. 
En las organizaciones, el desempeño constituye uno de los factores determinantes para el logro de las metas y objetivos de la institución, por aquello, es de gran importancia que los colaboradores mantengan una relación entre las competencias que poseen y el cargo que ejercen, para lo cual es necesario que se exija el cumplimiento de requisitos que realmente permitan la selección del personal con características que correspondan a la finalidad de la institución $^{(4)}$ (Figura 2).

Figura 2. Descripción del desempeño laboral. Fuente: Robbins (2010).

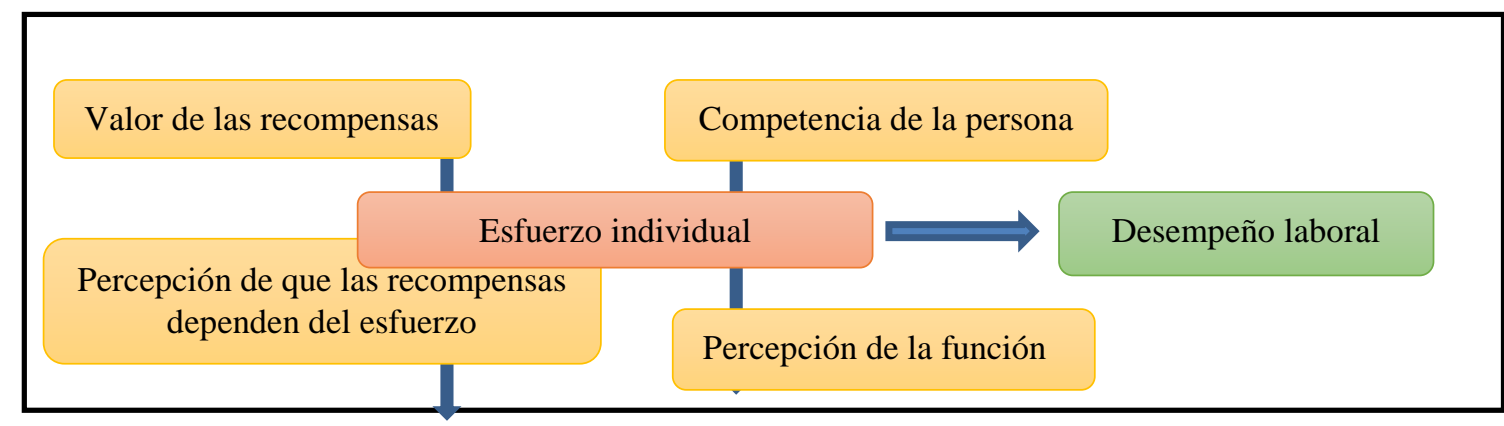

Uno de los aspectos de gran relevancia, que se debe tener en cuenta dentro de las organizaciones, es analizar el concepto de desempeño laboral, el cual en la evolución de la palabra ha sufrido cambio en su definición, sumando elementos que antes no eran relevantes, por ello diversos autores, han descrito y mencionado su propia definición de desempeño laboral $^{(1)}$ (Tabla 1).

Tabla 1. Evolución del concepto desempeño laboral.

\begin{tabular}{|l|c|l|}
\hline \multicolumn{2}{|c|}{} & \multicolumn{1}{|c|}{ Concepto de desempeño laboral } \\
\hline Autor & Año & \multicolumn{1}{c|}{ Definición } \\
\hline Sikula & 1989 & $\begin{array}{l}\text { Atribución de un valor que se da a cada actuación del empleado, } \\
\text { con el propósito de facilitar la toma de decisiones y lograr } \\
\text { resultados }\end{array}$ \\
\hline Chiavenato
\end{tabular}


Revisión desde el desempeño laboral al vínculo con Enfermería

\begin{tabular}{|l|c|l|}
\hline Lado & 2013 & $\begin{array}{l}\text { Establece que el desempeño va de la mano con la evaluación del } \\
\text { trabajo, el cual busca diferentes objetivos, tanto para la institución } \\
\text { como para los trabajadores; con el propósito de conocer si sus } \\
\text { comportamientos y resultados corresponden a las metas esperadas } \\
\text { por la organización, o bien si debería mejorarlos }{ }^{(9)} .\end{array}$ \\
\hline
\end{tabular}

Fuente: Elaboración propia, San Luis Potosí, 2021.

En la Tabla 1, se puede observar la evolución del concepto desempeño laboral, se ha ido complementando al pasar de los años, integrando nuevas aristas en la definición, ampliando el concepto. Actualmente existe una gran cantidad de criterios que podrían mencionarse al estimar el desempeño,

\section{Características del desempeño Laboral}

Las características del desempeño laboral corresponden a los conocimientos, habilidades y capacidades que se espera, quien aplique y demuestre al desarrollar su como las capacidades, habilidades, necesidades y cualidades son características individuales que interactúan con la naturaleza del trabajo y de la organización para producir comportamientos, el cual genera el desempeño laboral óptimo o desfavorable.

trabajo. Se refiere a la mantención de la efectividad en diferentes ambientes y con diferentes asignaciones, responsabilidades $\mathrm{y}$ personas, para ejercer su labor $\operatorname{asignada}^{(4)}$ (Tabla 2).

Tabla 2. Características del desempeño laboral.

Características del desempeño laboral

\begin{tabular}{|c|c|c|}
\hline Autor & Año & Postulado \\
\hline $\begin{array}{l}\text { Milkovich } \\
\text { y Boudrea }\end{array}$ & 1994 & $\begin{array}{l}\text { Mencionan que las mediciones de desempeño individual podrían } \\
\text { revelar el bajo desempeño al interior de las organizaciones, esto se } \\
\text { debe a una asistencia irregular en su labor o a una baja motivación al } \\
\text { trabajo que desempeña }{ }^{(10)} \text {. }\end{array}$ \\
\hline Chiavenato & 2000 & $\begin{array}{l}\text { Describe que las características individuales, entre las cuales se } \\
\text { pueden mencionar: las capacidades, habilidades, necesidades y } \\
\text { cualidades, entre otros, interactúan con la naturaleza del trabajo y de } \\
\text { la organización para producir un óptimo desempeño en los } \\
\text { trabajadores }^{(6)} \text {. }\end{array}$ \\
\hline $\begin{array}{c}\text { Davis y } \\
\text { Newtrons }\end{array}$ & 2000 & $\begin{array}{l}\text { Mencionan un listado de cualidades que los integrantes deberían } \\
\text { poseer, los cuales } \operatorname{son}^{(11)} \text { : } \\
\text { Capacidades. } \\
\text { Adaptabilidad. } \\
\text { Comunicación. } \\
\text { Iniciativa. } \\
\text { Conocimientos. }\end{array}$ \\
\hline
\end{tabular}


Trabajo en equipo.

Estándares de trabajo.

Desarrollo de talentos.

Potencia el diseño del trabajo.

Maximizar el desempeño.

Fuente: Elaboración propia, San Luis Potosí, 2021.

Las características vistas en la Tabla 2 del desempeño en los colaboradores, cobra cada vez más relevancia al ser de carácter individual, como lo mencionan diferentes autores. Las organizaciones están conscientes, de la importancia de considerar cuidadosamente estas dimensiones, durante el proceso de dirección estratégica en la organización de salud. Al mencionar las competencias humanas, se entiende que el colaborador integra cualidades de trabajo en equipo, conocimiento, formación, habilidades comunicacionales, entre otras. También competencia implica tener la capacidad de ejecución, destrezas intelectuales y psicomotoras para llevar a cabo la ejecución de su labor.

Harrington (2001), refiere que "El desempeño laboral comprende todas aquellas actividades y tareas, que realiza o ejecuta un determinado trabajador en una determinada empresa". Igualmente, el desempeño laboral aborda el cumpli- miento de sus tareas encomendadas de manera exitosa, teniendo una repercusión en las metas y los objetivos planeados por la organización ${ }^{(12)}$.

Chiavenato (2004), destaca "Las personas constituyen el recurso más valioso para las organizaciones. Por eso el efectivo desempeño del recurso humano constituye una de las claves de éxito de toda institución". Es necesaria la evaluación de los individuos que desempeñan los diferentes roles dentro de la organización, para determinar su aporte a la eficiencia organizacional ${ }^{(13)}$.

\section{Factores que influyen en el desempeño} laboral

Existen múltiples factores que influyen en el desempeño del trabajo. Puntualmente, cuando se habla de profesiones que brindan un servicio o están a cargo de la salud de una comunidad, estos factores se complejizan $^{(14)}$.

Tabla 3. Factores influyentes en el desempeño laboral.

\begin{tabular}{|c|c|l|}
\hline \multicolumn{2}{|c|}{ Factores que influyen al desempeño laboral } \\
\hline Autor & Año & \multicolumn{1}{c|}{ Postulado } \\
\hline Nash & 1988 & $\begin{array}{l}\text { Menciona diferentes factores a conocer }{ }^{(15)} \text { : } \\
\text { La percepción del empleado sobre la equidad, actitudes y opiniones } \\
\text { acerca de su trabajo. } \\
\text { El grado de importancia que tiene el trabajo para las personas que } \\
\text { lo ejecutan. } \\
\text { La autoestima como un sistema de necesidades del individuo, } \\
\text { dentro del grupo de trabajo. }\end{array}$ \\
\hline
\end{tabular}


Revisión desde el desempeño laboral al vínculo con Enfermería

\begin{tabular}{|c|c|c|}
\hline & & $\begin{array}{l}\text { La capacitación para trabajar la productividad. } \\
\text { La Remuneración como incentivo para mejorar la productividad. } \\
\text { La temperatura, en un estándar regulado de tal manera que caiga } \\
\text { dentro del rango aceptable del individuo. } \\
\text { El ruido, la mayoría de las oficinas deben operar en un rango de } \\
\text { bajo ha moderado. } \\
\text { La intensidad adecuada de iluminación según la dificultad de la } \\
\text { tarea y de la precisión requerida. } \\
\text { El tamaño, es el factor más importante para determinar cuánto } \\
\text { espacio se proporciona a un empleado. }\end{array}$ \\
\hline $\begin{array}{l}\text { Davis y } \\
\text { Newstrom }\end{array}$ & 2000 & $\begin{array}{l}\text { Mencionan que los factores influyentes se pueden dividir en } 4 \\
\text { categorías, las cuales son }{ }^{(11)} \text { : } \\
\text { Satisfacción del trabajo: Es el conjunto de sentimientos favorables } \\
\text { o desfavorables con los que el empleado percibe su trabajo, el cual } \\
\text { se manifiestan en determinadas actitudes laborales. } \\
\text { Autoestima: Sistema de necesidad del individuo, manifestando la } \\
\text { necesidad por lograr una nueva situación en la empresa, así como el } \\
\text { deseo de ser reconocido dentro del equipo de trabajo. } \\
\text { Trabajo en equipo: Labor realizada por los trabajadores en contacto } \\
\text { directo con los usuarios o a quienes presta el servicio, y/o su grupo } \\
\text { de trabajo. } \\
\text { Capacitación del trabajador. }\end{array}$ \\
\hline $\begin{array}{l}\text { Robbins y } \\
\text { Judge }\end{array}$ & 2013 & $\begin{array}{l}\text { Mencionan que las compañías actuales, menos jerárquicas y más } \\
\text { orientadas al servicio, requieren de más información hoy en día se } \\
\text { reconocen tres tipos principales de conductas que constituyen el } \\
\text { desempeño laboral }{ }^{(13)} \text { : } \\
\text { Desempeño de las tareas: Cumplimiento de las obligaciones y } \\
\text { responsabilidades que contribuyen a la producción de un bien o } \\
\text { servicio a la realización de las tareas administrativas. } \\
\text { Civismo: Acciones que contribuyen al ambiente psicológico de la } \\
\text { organización, como brindar ayuda a los demás, respaldar los } \\
\text { objetivos de la organización, respeto, hacer sugerencias } \\
\text { constructivas y decir cosas positivas sobre el lugar de trabajo. } \\
\text { Falta de productividad: Acciones que dañan de manera activa a la } \\
\text { organización (robo, daños a la propiedad de la compañía, } \\
\text { comportamiento agresivo y absentismo frecuente. }\end{array}$ \\
\hline
\end{tabular}

Fuente: Elaboración propia, San Luis Potosí, 2021.

Existen diversas fuentes que afectan el rendimiento como se observa en la Tabla 3, lo es, sobrecarga laboral, la falta de comunicación, el entorno laboral, la excesiva responsabilidad que conlleva el trabajo, las emociones, los propios pacientes y familiares en casos de organizaciones de salud, también los 
Jiménez Castro A, Echeverría Rivera S

conflictos con los médicos, entre otros. También pueden ser factores que inciden en el rendimiento, la recompensa económica, familiar, el bajo rendimiento de productividad y eficacia del trabajo.

El desempeño se vincula con las habilidades y conocimientos que apoyan las acciones del colaborador, en pro de consolidar los objetivos de la organización. La importancia de este enfoque, reside en el hecho que el desempeño del colaborador va de la mano con las actitudes y aptitudes que estos tengan, en función a los objetivos que se quieran alcanzar, seguidos por políticas normas, visión y misión de la organización ${ }^{(16)(14)}$.

\section{Elementos del desempeño laboral}

El desempeño laboral está determinado por factores actitudinales de la persona y factores operativos, los cuales son llamados elementos, estos influyen e interfieren el rendimiento de la organización. Los colaboradores deben poseer aspectos prácticos para poder efectuar un trabajo, esto permite perfeccionamiento el control de equipos y procedimientos (Tabla 4).

Tabla 4. Elemento del desempeño laboral según propuesta de diversos autores.

\begin{tabular}{|c|c|c|}
\hline \multicolumn{3}{|r|}{ Elementos del desempeño laboral } \\
\hline Autor & Año & Propuesta \\
\hline Stoner & 1994 & $\begin{array}{l}\text { Hace referencia a la Teoría de expectativa, se basa en cuatro } \\
\text { supuestos referentes al comportamiento organizacional: La } \\
\text { combinación de fuerzas en los individuos y el ambiente, las } \\
\text { decisiones conscientes sobre el comportamiento individual, las } \\
\text { distintas necesidades, deseos y metas de las personas, por último, } \\
\text { las opciones de comportamiento de las mismas, basándose en sus } \\
\text { expectativas }^{(4)} \text {. }\end{array}$ \\
\hline Ghiselli & 1998 & $\begin{array}{l}\text { El desempeño está influenciado por cuatro factores: la } \\
\text { motivación, habilidades, claridad y aceptación del rol. La } \\
\text { importancia de este enfoque reside en el hecho que el desempeño } \\
\text { del trabajador va de la mano con las actitudes y aptitudes que } \\
\text { estos tengan es función a los objetivos que se quieran } \\
\text { alcanzar }{ }^{(17)} \text {. }\end{array}$ \\
\hline Bittel & 2000 & $\begin{array}{l}\text { El desempeño laboral está determinado por: Factores } \\
\text { actitudinales (disciplina, actitud cooperativa, iniciativa, } \\
\text { responsabilidad, habilidad de seguridad, discreción, } \\
\text { presentación personal, interés, creatividad, capacidad de } \\
\text { realización), Factores operativos (conocimiento del trabajo, } \\
\text { calidad, cantidad, exactitud, trabajo en equipo, liderazgo) })^{(18)} \text {. }\end{array}$ \\
\hline $\begin{array}{l}\text { Arias } \\
\text { Galicia }\end{array}$ & 2001 & $\begin{array}{l}\text { Se refiere, a la administración del alto desempeño en la } \\
\text { aplicación de los procesos administrativos totales para lograr el } \\
\text { pleno florecimiento de las potencialidades humanas dentro de las } \\
\text { empresas u organizaciones }{ }^{(14)} \text {. }\end{array}$ \\
\hline
\end{tabular}


Revisión desde el desempeño laboral al vínculo con Enfermería

\begin{tabular}{|l|l|l|}
\hline Montenegro 2007 & $\begin{array}{l}\text { Es preciso tener claramente identificadas las acciones que serán } \\
\text { ejecutadas dentro de un ámbito delimitado. Luego de identificar } \\
\text { esas acciones, es necesario realizar la evaluación de este } \\
\text { desempeño para así establecer el grado eficiencia en la ejecución } \\
\text { de las acciones identificadas }\end{array}$ \\
\hline
\end{tabular}

Fuente: Elaboración propia, San Luis Potosí, 2021.

\section{Evaluación del desempeño}

La apreciación del desempeño en

un colaborador, es mediante un instrumento, el cual ayuda a dirigir y supervisar al personal. Entre sus principales objetivos se puede señalar el desarrollo personal y profesional de colaboración, la mejora permanente de resultados de la organización y el aprovechamiento adecuado de los recursos humanos. Por otro lado, la evaluación de desempeño debe realizarse siempre con relación al perfil de puesto que se requiere evaluar (Tabla 5).

Tabla 5. Evaluación del desempeño.

\begin{tabular}{|l|l|l|}
\hline \multirow{2}{*}{ Autor } & Año & \multicolumn{1}{|c|}{ Evaluación del desempeño } \\
\cline { 3 - 3 } Rondy y & 2005 & $\begin{array}{l}\text { Mencionan que la meta principal de un sistema de evaluación es } \\
\text { mejorar el desempeño individual y organizacional y este sistema } \\
\text { adecuadamente diseñado puede generar datos potencialmente } \\
\text { valiosos en la mayor parte de las áreas funcionales de recursos } \\
\text { humanos } \\
\text { Planeación de recursos humanos: Al evaluar los recursos humanos } \\
\text { de una empresa, debe generarse información que describa la } \\
\text { posibilidad de promoción y de los empleados basándose en su } \\
\text { potencial. } \\
\text { Reclutamiento y selección: Las calificaciones de la evaluación del } \\
\text { desempeño se pueden utilizar para predecir el desempeño de quienes } \\
\text { solicitan ser empleados. } \\
\text { Capacitación y Desarrollo: Una evaluación del desempeño debe } \\
\text { permitir detectar las necesidades específicas de capacitación y } \\
\text { desarrollo de un empleado. }\end{array}$ \\
\hline Chiavenato 2006 & $\begin{array}{l}\text { Describe y define dos puntos importantes a destacar, en los cuales } \\
\text { refiere } \\
\text { La evaluación del desempeño es "una apreciación sistemática de } \\
\text { cómo cada persona se desempeña en su puesto y de su potencial } \\
\text { de desarrollo futuro. Toda evaluación es un proceso para estimular, } \\
\text { la excelencia y las cualidades de una persona". } \\
\text { La evaluación del desempeño es importante en una organización, ya } \\
\text { que "es un medio por el cual se detectan problemas como es la } \\
\text { supervisión del personal, integración del empleado a la organización }\end{array}$ \\
\hline
\end{tabular}


Jiménez Castro A, Echeverría Rivera S

\begin{tabular}{|l|l|}
\hline & $\begin{array}{l}\text { y al puesto de trabajo, también desaprovechamiento de empleados } \\
\text { en puestos no adecuados a su potencial, así como también se puede } \\
\text { identificar si hay problemas de motivación". }\end{array}$ \\
\hline
\end{tabular}

Fuente: Elaboración propia, San Luis Potosí, 2021.

La evaluación del desempeño como se presenta en la Tabla 5, es un proceso formal de revisión y evaluación del rendimiento laboral individual o del equipo de trabajo. Es un procedimiento estructurado y sistemático para medir, evaluar e influir sobre los elementos, comportamientos y resultados relacionados con el trabajo de la organización ${ }^{(8)}$.

Existen programas de evaluación del desempeño, para conocer las capacidades e identificar el mérito respectivo, la evaluación no es novedad, actualmente toda empresa sea grande o pequeña, inclusive en los diferentes sectores público o privado la utilizan como herramienta para la medición del desempeño en sus colaboradores ${ }^{(21)}$.

\section{Dimensiones del desempeño laboral}

Las dimensiones se clasifican de acuerdo a las características, conductas o resultados. Los basados en característica son los más usados; los basados en conducta (competencias) brindan a los colaboradores información más orientada a la acción; mientras que el enfoque con base en resultados se focaliza en las contribuciones que los colaboradores realizan en la organización ${ }^{(21)}$.

\section{B. Búsqueda de estudios en desempeño laboral de Enfermería Enfermería y desempeño laboral}

Los autores Campos et al. (2018), en su estudio sobre desempeño laboral en profesionales de enfermería, mencionan en su investigación la relación que existe entre el profesional sanitario y su desempeño laboral. El cual, serviría para conocer los beneficios e impacto de realizar la gestión de recursos humanos, dejando en evidencia en sus resultados; ya que los profesionales de enfermería participantes, evidenciaron el tipo de desempeño profesional, describiéndolo como un desempeño moderado del 27,6\%, y solo un $17,2 \%$ un desempeño laboral alto al interior de la organización. También Quintana y Tarqui (2019), publicaron sus datos de investigación realizada en el Perú, donde ellos refieren que existen escasos estudios que aborden el desempeño del profesional de enfermería en establecimientos de salud, a pesar de que constituye un factor fundamental en la gestión y desarrollo de los hospitales debido a las implicancias que guarda la temática, la obtención de los resultados de la calidad de la atención y en particular de

la calidad humana, donde solo un $24,5 \%$ de los profesionales en enfermería presentó bajo desempeño, un 73,1\% medio desempeño y $2,4 \%$ alto desempeño ${ }^{(22)(23)}$.

\section{Medición del desempeño en Enfermería}

García y Cortez (2016), realizo la medición de desempeño laboral en colaboradores de salud, donde describe que los profesionales entrevistados comentan la necesidad de mecanismos de supervisión, apoyo técnico adecuado y 
Revisión desde el desempeño laboral al vínculo con Enfermería

retroalimentación, también de reconocimiento de los logros y de normas y códigos de conducta para proporcionar una mejor calidad de atención y mejorar el ambiente de trabajo. También describen, que, la administración del hospital donde se realizó el estudio se encuentra deteriorado y no se muestra interés por el desarrollo estratégico, que promuevan el empoderamiento entre los colaboradores $^{(24)}$.

\section{Factores predominantes en Enfermería}

Jara (2015), en la publicación de sus datos, menciona que existen factores predominantes de desempeño laboral, como la efectividad con un $46 \%$, que hace referencia al grado en que la organización es capaz de lograr sus metas. Por otro lado, el $30 \%$ presentan eficacia del desempeño laboral, que hace referencia a la proporción que refleja una comparación entre los resultados logrados y los costos surgidos para el cumplimiento de las $\operatorname{metas}^{(25)}$.

Jara también menciona en sus datos, el compromiso por parte de los colaboradores, indicando que no existe compromiso a obtener los resultados deseados por la organización en un $77 \%$, y solo un $25 \%$ opina que tratan de obtener los resultados deseados ${ }^{(25)}$.

En el trabajo realizado por Alejos (2017), menciona la importancia en las relaciones interpersonales, las cuales juegan un papel fundamental en el desarrollo integral de los colaboradores. A través de ellas, el individuo obtiene importantes refuerzos sociales del entorno laboral más inmediato, que favorecen su adaptación a la organización. Por otra parte, Arias (2013), concluyen que en las relaciones interpersonales de las enfermeras son imprescindible en una organización laboral basada en la solidaridad, autenticidad, autorrespeto, unidad, democracia y comunicación asertiva que favorezcan la convivencia, el cuidado humanizado y la resolución de conflictos laborales dentro del equipo de enfermería $^{(26)(25)}$.

\section{Características del desempeño en Enfermería}

Espinoza (2015), describe que el desempeño profesional de Enfermería que labora en el Primer Nivel de Salud, debe estar dotado, no solo de aquellos conocimientos y habilidades que favorezcan el desempeño profesional en su labor, sino de los valores éticos, la sensibilidad humana y el respeto a las personas que necesitan de los servicios de enfermería $^{(12)(27)}$.

Existe una gran cantidad de criterios que podrían medirse al estimar el desempeño. Las capacidades, habilidades, necesidades y cualidades son características individuales de cada colaborador en enfermería, el cual es un elemento intangible que interactúa con la naturaleza del trabajo y de la organización para producir comportamientos ${ }^{(2)}$.

\section{Adaptabilidad y desempeño laboral}

En la investigación realizada por Espinosa (2015), determinó según la prueba de correlación de Spearman que existe una relación significativa entre la inteligencia emocional; factor adaptabilidad: manejo del estrés; estado de ánimo y el desempeño laboral en el equipo de salud. Por otra parte, Pachas (2016), en su investigación reporta que el estrés se 
relaciona de manera inversa con el desempeño laboral, siendo un agente estresor para el equipo de enfermería, viendo reflejado en su desempeño dentro de la organización ${ }^{(27)}$.

\section{Evaluación del desempeño en Enfermería}

En la publicación de Díaz (2018), mencionan sus datos que la evaluación de desempeño, que corresponden a la autoevaluación, permite mostrar en el $78.5 \%$ un nivel regular en la dimensión calidad de trabajo, un $46.2 \%$ nivel bueno en la dimensión estabilidad emotiva y solo un $20 \%$ nivel deficiente en la dimensión capacidad analítica ${ }^{(28)}$.

\section{DISCUSIÓN}

A través de esta revisión, se ha podido conocer cómo se relaciona el desempeño laboral con el profesional de enfermería, además de conocer los conceptos, fundamentos, dimensiones $\mathrm{y}$ características que tiene el desempeño laboral según las propuestas de diversos autores de literatura en temáticas de administración y el manejo del recurso humano. Todas las áreas de desarrollo el desempeño laboral y su importancia en el capital humano de las organizaciones, se ven reflejados de igual manera en los profesionales de enfermería, y más aún, por la importancia que tiene en la atención directa con los usuarios en todos los niveles de atención en salud.

Por ello, es importante realizar más investigaciones con respecto a la temática para ver el desempeño de la profesión de enfermería, así ir mejorando las áreas de deficiencia, la cual ha de ir, evolucionando en las áreas de administración del capital humano en salud.

\section{CONCLUSIONES}

Conocer el nivel el desempeño laboral del profesional de enfermería y las áreas que abarcan los conceptos teóricos en la literatura en administración y recurso humano, entrega mayores competencias, formación continua, y mayor profesionalismo (autonomía, comunicación y participación en organizaciones sanitarias). De esta forma, ayuda a establecer estrategias de abordaje para conocer las falencias del actuar en enfermería, realizar un análisis del contexto en donde se desarrolla el fenómeno en los profesionales y poder entregar una solución, mejorando el desempeño del profesional o del equipo de salud.

En este sentido, esta revisión es un aporte a la disciplina, al exponer los conceptos de un área administrativa y de recursos humanos, los cuales se relacionan con los estudios realizados en desempeño de enfermería, observando los hallazgos de lo encontrado por diversos autores; por lo tanto, se espera motivar una discusión al respecto orientada a una mejora continua, y mayor investigación en la temática, así conocer de mejor forma el fenómeno de rendimiento laboral en enfermería.

El profesional de enfermería debe avanzar en el logro de las exigencias sanitarias, a través plena autonomía profesional, publicar y comunicar los resultados de sus investigaciones, lo que a su vez le va a otorgar autonomía por los conocimientos que esto implica, y participar activamente en organizaciones profesionales. Desde otra mirada, esta 
Revisión desde el desempeño laboral al vínculo con Enfermería

revisión es un aporte al fortalecimiento de la investigación de enfermería, es un primer paso que evidencia esta problemática, que se convierte en un sustento para trabajos posteriores que profundicen en este ámbito y evalúen el desempeño laboral como una competencia.

\section{REFERENCIAS BIBLIOGRÁFICAS}

1. Minsal D, Pérez Y. Hacia una nueva cultura organizacional: La cultura del conocimiento. Acimed. 2007;16(3).

2. Revilla D, Santana R, Rentería M. La influencia de la cultura organizacional en el desempeño hospitalario. Rev Sanid Milit. 2015;69(5):429-39.

3. Robbins R, Stephen P, Coulter M. Administración. 10ª Edició. Educación P, editor. México; 2010.

4. Robbins S, Judge T. Comportamiento organizacional: conceptos, controversias y aplicaciones. 6 edición. México: Prentice-Hall Hispanoamericana; 1994.

5. Sikula A, McKenna J. Administración de recursos humanos: Conceptos prácticos. Limusa. 1989.

6. Chiavenato I. Administración de recursos humanos. Quinta edi. Loa Angeles: Mg Graw Hill; 2000.

7. Harrington J. Health effects of shift work and extended hours of work. Occup Environ Med. 2001;58(1):6872.

8. Sánchez J, Bustamante K. Auditoría al proceso de evaluación del desempeño. Madrid; 2008. 105-133 p.

9. Lado M. Introducción a la Auditoría Sociolaboral: una perspectiva desde los Recursos Humanos. Bubok Publ.
España; 2013.

10. Milkovich G, Boudreau J. Dirección y Administración de recursos humanos: Un enfoque de estrategia. Addison-We. Estados Unidos; 1994.

11. Davis K, Newtrons J. Comportamiento Humano en el Trabajo. Décima Edi. México: McGraw - Hill; 2000.

12. Diez E. La organización educativa, estrategias de análisis e innovación. Ediversita. Sevilla; 2001.

13. Robbins S, Judge T. Comportamiento organizacional. 15 edición. México: Pearson Educación; 2013.

14. Arias F. Administración de recursos humanos para el alto desempeño. Sexta edic. México: Editorial Trillas; 2001.

15. Michael N. Cómo incrementar la productividad del recurso humano: mediante técnicas que abarcan toda la relación laboral. Cuarta edi. Colombia: Norma; 1988.

16. Montenegro A, AbdónI G. Evaluación del desempeo docente fundamentos modelos e instrumentos. Colombia: Magisterio; 2007.

17. Ghiselli M. Administración de Personal. Traducción José Real Gutiérrez, editor. 1998.

18. Bitte L. Administración de Personal. Colombia: Mc Graw Hill; 2000.

19. Mondy W, Robert N. Administracion de recursos humanos. 9 edición. México: Pearson; 2005.

20. Chiavenato J. Introducción a la teoría general de la administración. McGrawHill, editor. Vol. 7a. edició. México; 2006. p. 13.

21. Capuano A. Evaluación desempeño por Competencias. Invenio [Internet]. 2004;7(13):139-50. Available from: http://www.redalyc.org/pdf/877/87713 
710.pdf

22. Campos P, Gutiérrez H, Matzumura J. Rotación y desempeño laboral de los profesionales de enfermería en un instituto especializado. Rev Cuid [Internet]. 2019 May 3;10(2):564-8. Available from: http://eds.a.ebscohost.com/eds/detail/d etail?sid=0ae9faf3-4ba5-4a56-b715bb4681b84ac5@sessionmgr4003\&vid $=0 \&$ hid $=4103 \&$ bdata $=$ Jmxhbmc9cHQ tYnImc210ZT11ZHMtbG12ZSZzY29w ZT1zaXRl\#db=lth\&AN=109999041

23. Tarqui C, Quintana D. Desempeño laboral del profesional de enfermería en un hospital de la Seguridad Social del Callao - Perú. Arch Med [Internet]. 2019 Dec 15;20(1):123-32. Available from:

http://revistasum.umanizales.edu.co/oj s/index.php/archivosmedicina/article/v iew/3372/5439

24. Vegro T, Rocha F, Camelo S, Garcia A. Cultura organizacional de um hospital privado. Rev Gaúcha Enferm [Internet]. 2016;37(2):e49776. Available from: http://www.scielo.br/scielo.php?script =sci_arttext\&pid=S1983$14472016000200401 \& \operatorname{lng}=$ pt\&tlng=pt

25. Jara L, Juárez J. Relaciones Interpersonales y Desempeño Laboral en los trabajadores del Centro de Salud Morales, 2015. Rev Peru Obstet y Enfermería [Internet]. 2015;4(1):1-8. Available from: http://www.ncbi.nlm.nih.gov/pubmed/ 17289255\%0Ahttp://dx.doi.org/10.101 6/j.jclepro.2011.10.008\%0Ahttp://ww w.uwaba.or.tz/National_Road_Safety_ Policy_September_2009.pdf\%0Ahttp:/ /dx.doi.org/10.1016/j.resconrec.2011.0
Jiménez Castro A, Echeverría Rivera S

3.010\%0Ahttp://dx.doi.org/10.1016/j.p ecs. 20

26. Lorena M, Padilla S, Alcántara S. Salud del personal de enfermería del hospital general de México 2007. 2019;(1).

27. Espinoza O. La inteligencia emocional y el desempeño por competencias de docentes en la I.E. Mentes Brillantes Trujillo, 2014. UCV - HACER Rev Investig y Cult [Internet]. 2015;4(2):18-24. Available from: http://www.redalyc.org/articulo.oa?id= 521751974002

28. Díaz C, Gutiérrez H, Amancio A. Ausentismo y desempeño laboral en profesionales de enfermería de áreas críticas. Rev Cuid. 2018;9(1):1973. 